\title{
A NEW METHOD TO CONSTRAIN SUPERNOVA FRACTIONS USING X-RAY OBSERVATIONS OF CLUSTERS OF GALAXIES
}

\author{
EsRa Bulbul ${ }^{1,2}$, Randall K. SMith ${ }^{1}$, and Michael Loewenstein ${ }^{2,3}$ \\ ${ }^{1}$ Harvard-Smithsonian Center for Astrophysics, 60 Garden Street, Cambridge, MA 02138, USA; ebulbul@cfa.harvard.edu \\ ${ }^{2}$ CRESST and X-Ray Astrophysics Laboratory, NASA/GSFC, Greenbelt, MD 20771, USA \\ ${ }^{3}$ Department of Astronomy, University of Maryland, College Park, MD 20742, USA \\ Received 2012 February 23; accepted 2012 April 26; published 2012 June 13
}

\begin{abstract}
Supernova (SN) explosions enrich the intracluster medium (ICM) both by creating and dispersing metals. We introduce a method to measure the number of SNe and relative contribution of Type Ia supernovae ( $\mathrm{SNe} \mathrm{Ia}$ ) and core-collapse supernovae ( $\mathrm{SNe}$ cc) by directly fitting X-ray spectral observations. The method has been implemented as an XSPEC model called snapec. snapec utilizes a single-temperature thermal plasma code (apec) to model the spectral emission based on metal abundances calculated using the latest SN yields from SN Ia and SN cc explosion models. This approach provides a self-consistent single set of uncertainties on the total number of SN explosions and relative fraction of SN types in the ICM over the cluster lifetime by directly allowing these parameters to be determined by SN yields provided by simulations. We apply our approach to XMM-Newton European Photon Imaging Camera (EPIC), Reflection Grating Spectrometer (RGS), and $200 \mathrm{ks}$ simulated Astro-H observations of a cooling flow cluster, A3112. We find that various sets of SN yields present in the literature produce an acceptable fit to the EPIC and RGS spectra of A3112. We infer that $30.3 \% \pm 5.4 \%$ to $37.1 \% \pm 7.1 \%$ of the total SN explosions are $\mathrm{SNe}$ Ia, and the total number of $\mathrm{SN}$ explosions required to create the observed metals is in the range of $(1.06 \pm 0.34) \times 10^{9}$ to $(1.28 \pm 0.43) \times 10^{9}$, from snapec fits to RGS spectra. These values may be compared to the enrichment expected based on well-established empirically measured SN rates per star formed. The proportions of SNe Ia and SNe cc inferred to have enriched the ICM in the inner $52 \mathrm{kpc}$ of A3112 is consistent with these specific rates, if one applies a correction for the metals locked up in stars. At the same time, the inferred level of SN enrichment corresponds to a star-to-gas mass ratio that is several times greater than the $10 \%$ estimated globally for clusters in the A3112 mass range.
\end{abstract}

Key words: galaxies: clusters: intracluster medium - galaxies: individual (A3112) - nuclear reactions, nucleosynthesis, abundances - supernovae: general - X-rays: galaxies: clusters

Online-only material: color figure

\section{INTRODUCTION}

The use of Type Ia supernovae ( $\mathrm{SNe}$ Ia) as standardizable candles in the discovery of dark energy (Riess et al. 1998; Perlmutter et al. 1999) installed the nature of SN Ia progenitors and the physics of the explosion as one of the prime problems in astrophysics. The evolution of the SN Ia rate is one of the promising methods for unveiling SN Ia progenitors (Ruiz-Lapuente \& Canal 1998; Yungelson \& Livio 2000). Measuring the evolution of the supernova ( $\mathrm{SN}$ ) rate is particularly important at higher redshifts where direct SN rate constraints are limited (Gal-Yam et al. 2002; Barbary et al. 2012).

Galaxy clusters represent the largest scales of organized matter in the universe, making them unique laboratories for chemical enrichment of the universe from all possible sources including SNe Ia. The intracluster medium (ICM) emits X-rays due to the highly ionized gas which has been heated by infall from the intergalactic medium (Gunn \& Gott 1972). The metals produced, during all stages of cluster formation and evolution, by stars and galaxies enter the ICM via SN explosions (De Young 1978) and strong galactic winds (Mathews \& Baker 1971). They remain in the ICM due to the cluster's deep potential well. Since X-ray spectroscopy yields accurate measurements of metal abundances in the ICM, the large reservoir of metals in clusters of galaxies provides a unique way to probe $\mathrm{SN}$ rates and therefore SN Ia progenitor models on a universal scale.

The launch of ASCA triggered studies of the relative contributions of SNe Ia and core-collapse supernovae ( $\mathrm{SNe} c \mathrm{cc}$ ) to
ICM metal enrichment. These studies used specific elemental abundance ratios (e.g., nickel to iron) measured in clusters to distinguish between SN Ia models (Baumgartner et al. 2005; Dupke \& White 2000; Dupke \& Arnaud 2001; Mushotzky \& Loewenstein 1997). With the launch of observatories with better spectral and spatial resolution, such as XMM-Newton, Chandra, and Suzaku, radial profiles of specific elemental abundances were used to determine the relative contribution of SNe Ia to the enrichment of clusters as a function of position (e.g., Matsushita et al. 2007; Sato et al. 2007; Million et al. 2011). High-resolution XMM-Newton Reflection Grating Spectrometer (RGS) measurements of specific elemental abundance ratios within the ICM have also been used to determine the integral yield of all the $\mathrm{SN}$ Ia and SN cc explosions and to distinguish between SN models with different levels of pre-enrichment of the progenitors and with different initial mass functions (IMF; Werner et al. 2006b; de Plaa et al. 2007; Grange et al. 2011).

In this paper we introduce a new approach to determine the total number of SN explosions, and the relative contributions of different SN types, to the total enrichment of the ICM using high-resolution X-ray observations. The method relies on using the full set of nucleosynthetic yields produced by recent SN models for elements with emission lines in the $\mathrm{X}$-ray bandpass to probe the total number of SN explosions and relative contribution of SNe Ia. We validate this approach using CCD resolution XMM-Newton European Photon Imaging Camera (EPIC) and high-resolution RGS observations of the cooling flow cluster A3112. A brief summary of the method is 
given in Section 2. The application of the method to analysis of XMM-Newton RGS, EPIC, and simulated Astro-H Soft X-Ray Spectrometer (SXS) data is outlined in Section 3. We provide our discussions and conclusions in Sections 4 and 5.

\section{XSPEC SUPERNOVA ABUNDANCE MODEL (snapec)}

\subsection{A Brief Description of snapec}

Most of the metals from oxygen through nickel residing in the ICM were synthesized by SN Ia or SN cc explosions. We provide a new XSPEC model called snapec to determine the total number of $\mathrm{SN}$ explosions and relative contributions of SN types to the metal content of the ICM using elemental yields provided by $\mathrm{SN}$ nucleosynthesis calculations in the literature. The expected elemental yields of SNe Ia and SNe cc from massive (10-50 $\left.M_{\odot}\right)$ progenitor stars of various metallicity $\left(0-1 A_{\odot}\right)$ have been intensively studied by several authors (Woosley \& Weaver 1995; Iwamoto et al. 1999; Nomoto et al. 2006). For instance, SN models predict that $\mathrm{SNe}$ Ia produce significant amounts of iron $(\mathrm{Fe})$, nickel $(\mathrm{Ni})$, and silicon $(\mathrm{Si})$, while, for $\mathrm{SNe} c \mathrm{c}$, large quantities of oxygen $(\mathrm{O})$, neon $(\mathrm{Ne})$, and magnesium $(\mathrm{Mg})$ are produced but very little $\mathrm{Fe}$ and $\mathrm{Ni}$ escapes the compact remnant.

snapec calculates the mass of the $i$ th element $\left(M_{i}^{\mathrm{SNe}}\right)$ in terms of the number of SN Ia $\left(N^{\mathrm{Ia}}\right)$ and $\mathrm{SN} \mathrm{cc}\left(N^{\mathrm{cc}}\right)$ explosions that enrich the ICM and the yields per SNe Ia $\left(y_{i}^{\mathrm{Ia}}\right)$ and $\mathrm{SNe} \operatorname{cc}\left(y_{i}^{\mathrm{cc}}\right)$,

$$
\begin{gathered}
M_{i}^{\mathrm{SNe}}=N^{\mathrm{Ia}} y_{i}^{\mathrm{Ia}}+N^{\mathrm{cc}}\left\langle y_{i}^{\mathrm{cc}}\right\rangle \\
=N^{\mathrm{SNe}}(1+R)^{-1}\left[R y_{i}^{\mathrm{Ia}}+\left\langle y_{i}^{\mathrm{cc}}\right\rangle\right],
\end{gathered}
$$

where $R$ is the ratio of SNe Ia to $\mathrm{SNe}$ cc $\left(R=N^{\mathrm{Ia}} / N^{\mathrm{cc}}\right)$, and $N^{\mathrm{SNe}}$ is the total number of SN explosions $N^{\mathrm{SNe}}=N^{\mathrm{Ia}}+N^{\mathrm{cc}}$. The SN yields $y_{i}^{\mathrm{Ia}}$ and $y_{i}^{\mathrm{cc}}$ are obtained from published SN models and stored in the snapec database in solar units. The database includes yields of 30 elements from SN Ia explosions of slow deflagration (W7, C-DEF), delayed detonation (WDD, CDDT, ODDT), and core degenerate scenarios (CDD) (Tsujimoto et al. 1995, hereafter T95; Iwamoto et al. 1999, hereafter I99; Maeda et al. 2010, hereafter M10), and from SN cc yields from an extensive range of progenitor masses $\left(10-50 M_{\odot}\right)$ and metallicities (0-1 times solar) (Tsujimoto et al. 1995; Iwamoto et al. 1999; Woosley \& Weaver 1995, hereafter WW95; Nomoto et al. 2006, hereafter N06). snapec adopts solar abundances for metals with atomic number $\leqslant 7$ (e.g., helium, lithium, beryllium, boron, carbon, and nitrogen).

The snapec model allows a selection of solar abundance standards - the database incorporates the sets of Anders \& Grevesse (1989), Lodders (2003), or Asplund et al. (2009). Since the majority of hydrogen and helium in the ICM was produced during the big bang, the standard hot big bang nucleosynthesis (BBN) model predicts the primordial helium abundance by unit mass $Y_{P}$ to be $0.2565 \pm 0.0010$ (Izotov \& Thuan 2010) which corresponds to $8.3 \%$ helium atoms in number assuming 0.3 solar abundances for metals (e.g., Markevitch 2007). The Asplund et al. (2009) solar abundances we use here predict $8.5 \%$ of helium which is consistent with BBN predictions.

The SN cc yields of the $i$ th element, averaged over the IMF $(\phi)$, is

$$
\left\langle y_{i}^{\mathrm{cc}}\right\rangle=\frac{\int_{m_{1}}^{m_{2}} y_{i}^{\mathrm{cc}}(m) \phi(m) d m}{\int_{m_{1}}^{m_{2}} \phi(m) d m},
$$

where $m_{1}$ and $m_{2}$ are the lower and upper limits for the masses of SN cc progenitors. Here we assume that stars with masses
$M \leqslant 10 M_{\odot}$ and $M \geqslant 50 M_{\odot}$ do not explode as SNe cc. In this work we use the standard Salpeter IMF slope above $10 M_{\odot}$, $\phi=m^{-2.35}$ (Salpeter 1955).

It is straightforward to transform Equation (2) to an expression for the abundance, by number relative to hydrogen, of the $i$ th element. As long as the total mass fraction of metals is small and the $\mathrm{He}$ abundance is solar,

$$
\begin{gathered}
\frac{Z_{i}^{\mathrm{SNe}}}{Z_{i}^{\odot}}=\frac{M_{i}^{\mathrm{SNe}}}{M_{i}^{\odot}} \\
=\frac{N^{\mathrm{SNe}}}{M_{i}^{\odot}(1+R)}\left[R y_{i}^{\mathrm{Ia}}+\left\langle y_{i}^{\mathrm{cc}}\right\rangle\right] .
\end{gathered}
$$

$M_{i}^{\odot}$ is the mass of the $i$ th element for an ICM with solar abundances, i.e.,

$$
M_{i}^{\odot} \approx \frac{M_{\mathrm{ICM}} Z_{i}^{\odot} A_{i}}{m_{N}},
$$

where $Z_{i}^{\odot}$ is the abundance of the $i$ th element in solar units, $A_{i}$ is the atomic mass of the $i$ th element, and $m_{N}=2.27 \times 10^{-24}$ $\mathrm{g} / \mathrm{H}$-atom is the average nucleon mass per hydrogen atom (Asplund et al. 2009).

This XSPEC model, snapec, has five free parameters: gas temperature $\left(k T_{e}\right)$, the total number of $\mathrm{SN}$ explosions $\left(N^{\mathrm{SNe}}\right)$ and the $\mathrm{SN}$ ratio $(R)$ that enriches the ICM in the spectral region being analyzed, the cluster's redshift, and the XSPEC normalization factor identical to those defined for other thermal plasma models (see below). In addition, there are two fixed parameters, numerical indices that specify the SN Ia and SN cc models from which the yields are drawn. The predicted spectrum is constructed using the single-temperature thermal model (apec) (Smith et al. 2001) and AtomDB 2.0.1 (A. Foster et al. 2012, in preparation) to determine the continuum and line emission for the metal abundances calculated using Equation (5). This method has the advantage of simultaneously providing a single set of uncertainties on the $\mathrm{SN}$ ratio and the total number of SN explosions that enrich the ICM since the formation of the cluster. The results presented in this work are obtained using the Asplund et al. (2009) solar abundances.

snapec redefines the quantities in Equation (5) to express the abundances in terms the total number of SNe per $10^{12} M_{\odot}$ of ICM plasma-i.e., rescaled to yield values appropriate for cluster cores. The total number of $\mathrm{SNe}$ is derived by multiplying by the ICM mass (in units of $10^{12} M_{\odot}$ ) within the spectral extraction region. This may be estimated from the XSPEC normalization, which is proportional to the emission measure,

$$
N=\frac{10^{-14}}{4 \pi(1+z)^{2} D_{A}^{2}} \int n_{e} n_{\mathrm{H}} d V,
$$

where $n_{e}$ is the electron number density, $D_{A}$ is the angular diameter distance, and $z$ is the cluster's redshift.

\section{APPLICATIONS TO X-RAY SPECTRA}

Accurately determining the elemental abundances is crucial for understanding the relative contributions of different $\mathrm{SN}$ types to the metal enrichment of galaxies and intracluster gas. Earlier attempts to determine the relative fraction of $\mathrm{SNe}$ Ia using XMM-Newton measurements of individual elements such as $\mathrm{Si}$, $\mathrm{S}$, Ar, Ca, Fe, and Ni in the ICM (Werner et al. 2006b; de 
Table 1

XMM-Newton RGS Observations of A3112

\begin{tabular}{lcccr}
\hline \hline Obs. ID & R.A. & Decl. & $\begin{array}{c}\text { Exposure } \\
\text { Time } \\
(\mathrm{ks})\end{array}$ & $\begin{array}{c}\text { Clean } \\
\text { Time } \\
(\mathrm{ks})\end{array}$ \\
\hline 0603050101 & 031757.4 & -441414.7 & 118.9 & 100.1 \\
0603050201 & 031757.4 & -441412.8 & 80.8 & 80.4 \\
\hline
\end{tabular}

Plaa et al. 2007) concluded that the T95 and I99 SN models overestimated $\mathrm{Ar}$ and $\mathrm{Ca}$ abundances, and could not produce acceptable fits to XMM-Newton data. de Plaa et al. (2007) also inferred a number ratio of $\mathrm{SNe}$ cc to $\mathrm{SNe}$ Ia (using W7 yields) of $\sim 3.5$, based on XMM-Newton observations of 22 clusters. Grange et al. (2011) studied EPIC and RGS observations of two groups of galaxies, NGC 5044 and NGC 5813, and found that $\sim 30 \%-40 \%$ of $\mathrm{SNe}$ contributing to metal enrichment of the intragalactic gas were Type Ia while the other $\sim 60 \%-70 \%$ of $\mathrm{SNe}$ then were core collapse. This result was consistent with what was previously found for M87 by Werner et al. (2006a). Simionescu et al. (2009) reported that WDD3 or W7 $\mathrm{SN}$ Ia models are needed in order to reproduce the low $\mathrm{Si}$ abundance in Hydra A clusters of galaxies. They also noted that abundance patterns in the XMM-Newton observation of all other clusters preferred WDD1 and WDD2 models. However, these studies proceeded by first determining the best-fit values and uncertainties on individual elemental abundances, followed separately by fitting abundance ratios to determine the relative fractions of SNe Ia and SNe cc using the T95 and I99 models. By contrast, in this work we apply the yields obtained from $\mathrm{SN}$ models to immediately predict the spectrum, skipping the intermediate step required in previous studies. The best-fit $R$ and $N^{\mathrm{SNe}}$ parameters, and parameter confidence levels, are thus directly estimated from X-ray spectra and their statistical uncertainties for a given set of SN yields, allowing one to use the goodness of fit to assess the accuracy of the predictions and compare different yield sets on a well-defined statistical basis.

\subsection{XMM-Newton RGS Observations of A3112}

XMM-Newton's high spectral resolution RGS instrument (wavelength bandpass ranging from 5 to $38 \AA$ ) can resolve strong $\mathrm{X}$-ray lines and measure elemental abundances accurately in the compact central regions of nearby bright clusters of galaxies. We examined the RGS observations of the cool core cluster A3112 (see Table 1) using version 11.0 of the Science Analysis
Subsystem (SAS) software. For details about RGS data reduction and analysis, see Bulbul et al. (2012, hereafter Paper I). We fit the combined RGS first- and second-order spectra using an absorbed snapec model in the 7-22 $\AA$ and 7-16 $\AA$ wavelength range, respectively. The absorption column density was fixed to the Leiden/Argentine/Bonn Galactic value (Kalberla et al. $2005)$. The $N^{\mathrm{SNe}}, R$, redshift, $k T_{e}$, and normalization parameters were allowed to freely vary. The best-fit model parameters obtained from the snapec fits to RGS spectra for various SN models are shown in Table 2. Since, as in Paper I, fitting is conducted using the $C$-statistic and the $C$-statistic does not provide a direct estimate of the goodness of fit, we asses the goodness of fit using the corresponding $\chi^{2}$ value. These final $\chi^{2}$ values, obtained from the best fit determined by $C$-statistics, are reported in Table 2.

We calculated the projected gas mass of A3112 confined in the central 38" region corresponding to the effective RGS aperture (Paper I) based on the best-fit normalization parameter in the single-temperature thermal model (apec) fit to the RGS spectra using Equation (7). The apec normalization of $1.28 \times 10^{-2}$ gives a projected gas mass of $(4.80 \pm 0.70) \times 10^{11} M_{\odot}$ within the $38^{\prime \prime}(\sim 52 \mathrm{kpc})$ region. We also calculated a spherical gas mass of $(4.06 \pm 0.43) \times 10^{11} M_{\odot}$ within the $38^{\prime \prime}(\sim 52 \mathrm{kpc})$ which is consistent with the projected mass obtained from the apec normalization. This spherical gas mass was calculated using the deprojection analysis and modeling of the XMM-Newton EPIC observations of A3112 (see Bulbul et al. 2010, 2012). Thus, a conversion factor 0.41 is applied to the $\mathrm{SNe}$ per $10^{12} M_{\odot}$ derived from spectral fits to obtain the total number of SNe, $N^{\mathrm{SNe}}$ parameters, which are also reported in Table 2.

We also convert the $R$ parameter obtained from snapec fits into the fractional contribution of $\mathrm{SNe}$ Ia to the total number of SN explosions as follows:

$$
\begin{gathered}
R_{\%}^{\mathrm{Ia}}=\frac{\mathrm{SNe} \mathrm{Ia}}{(\mathrm{SNe} \mathrm{Ia}+\mathrm{SNe} \mathrm{cc})} \\
=\frac{R}{1+R} .
\end{gathered}
$$

We find that $30.3 \% \pm 5.4 \%$ to $37.1 \% \pm 7.1 \%$ of the total $\mathrm{SN}$ explosions are SNe Ia for models that use W7, CDD, and WDD yields. Application of M10 CDDT and CDEF SN Ia models implies a significantly higher fraction of SN Ia explosions $(\sim 57 \% \pm 14.5 \%)$. The reason for this difference is that M10

Table 2

\begin{tabular}{|c|c|c|c|c|c|c|c|}
\hline $\begin{array}{l}\text { SNe Ia } \\
\text { Model }\end{array}$ & $\begin{array}{l}\text { SNe cc } \\
\text { Model }\end{array}$ & $\begin{array}{c}k T_{e} \\
(\mathrm{keV})\end{array}$ & $\begin{array}{c}N^{\mathrm{SNe}} \\
\left(\times 10^{9}\right)\end{array}$ & $R$ & $\begin{array}{l}R_{\%}^{\mathrm{Ia}} \\
(\%)\end{array}$ & $\begin{array}{c}\chi^{2} \text { of Source } \\
\text { Spectra } \\
(2608 \text { dof })\end{array}$ & $\begin{array}{c}\chi^{2} \text { of Bkgd } \\
\text { Spectra } \\
(1490 \text { dof })\end{array}$ \\
\hline I99 (W7) & I99 & $3.70 \pm 0.23$ & $1.06 \pm 0.34$ & $0.52 \pm 0.09$ & $34.2 \pm 6.3$ & 2818.7 & 2026.4 \\
\hline I99 (W70) & I99 & $3.74 \pm 0.23$ & $1.07 \pm 0.33$ & $0.50 \pm 0.09$ & $33.5 \pm 6.2$ & 2822.5 & 2025.5 \\
\hline I99 (WDD1) & I99 & $3.81 \pm 0.23$ & $1.15 \pm 0.35$ & $0.57 \pm 0.10$ & $36.2 \pm 6.8$ & 2835.8 & 2022.9 \\
\hline I99 (WDD3) & I99 & $3.80 \pm 0.21$ & $1.06 \pm 0.33$ & $0.44 \pm 0.08$ & $30.3 \pm 5.4$ & 2829.5 & 2024.1 \\
\hline I99 (CDD1) & I99 & $3.81 \pm 0.22$ & $1.17 \pm 0.35$ & $0.59 \pm 0.12$ & $37.1 \pm 7.1$ & 2836.6 & 2022.8 \\
\hline I99 (CDD2) & I99 & $3.81 \pm 0.22$ & $1.08 \pm 0.34$ & $0.45 \pm 0.08$ & $31.2 \pm 5.7$ & 2831.5 & 2023.7 \\
\hline M10 (W7) & I99 & $3.70 \pm 0.23$ & $1.06 \pm 0.33$ & $0.51 \pm 0.09$ & $33.9 \pm 6.4$ & 2819.2 & 2826.2 \\
\hline M10 (CDEF) & I99 & $3.66 \pm 0.30$ & $1.28 \pm 0.43$ & $1.23 \pm 0.27$ & $56.3 \pm 13.7$ & 2809.5 & 2026.9 \\
\hline M10 (CDDT) & I99 & $3.33 \pm 0.23$ & $1.17 \pm 0.41$ & $1.34 \pm 0.29$ & $57.2 \pm 14.5$ & 2806.4 & 2026.8 \\
\hline M10 (ODDT) & I99 & $3.71 \pm 0.23$ & $1.12 \pm 0.34$ & $0.61 \pm 0.11$ & $37.8 \pm 7.2$ & 2823.6 & 2025.1 \\
\hline
\end{tabular}

The Best-fit Parameters of the snapec Model Obtained Using T95, I99, and M10 SN Yields and XMM-Newton RGS Spectra 


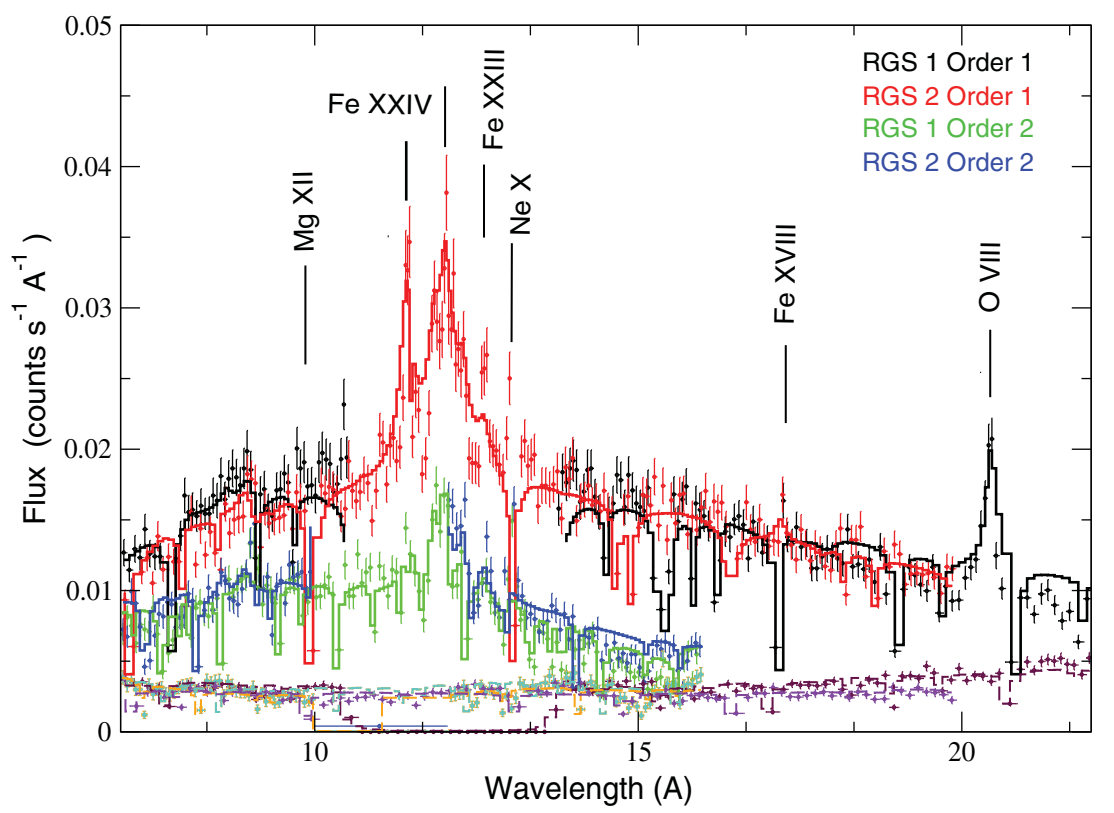

Figure 1. First- and second-order RGS sources and background spectral fits using the snapec model. The fit was performed using Iwamoto et al. (1999) SN Ia yields obtained from W7 models and SN cc yields obtained from the weighted average over a Salpeter IMF. The best-fit temperature, total number of supernovae, and SN Ia percentage of the total SNe are $3.69 \pm 0.23 \mathrm{keV},(1.24 \pm 0.33) \times 10^{9}$, and $34.3 \% \pm 6.4 \%$, respectively (Table 2). The total $\chi^{2}$ is 2818.7 for $2615 \mathrm{degrees}$ of freedom for the best fit to the source spectra, and 2026.4 for 1498 degrees of freedom for the background fit. (Table 2).

CDDT, CDEF SN Ia models have lower Fe abundance compared to the W7, W70, CDD, WDD, and ODDT models. Since Fe is mainly produced by SN Ia explosions, a lower abundance of $\mathrm{Fe}$ in SN Ia models in M10 CDDT, CDEF yields results in a higher number of SN Ia explosions and thus higher SN Ia fraction. The fraction of SN Ia explosions inferred using the I99 CDD and WDD models reported in Table 2 are consistent with those in de Plaa et al. (2007) based on elements accessible to the EPIC detectors: Si, S, Ar, Ca, Fe, and Ni. However, de Plaa et al. (2007) find a lower SN Ia fraction for W7 SN Ia models.

The SN models we use in this work produce equally good fits to RGS data (see Table 2). Therefore, global fits to the currently available high-resolution X-ray observations of clusters of galaxies do not allow one to distinguish between different types of SN models in an individual cluster. The RGS first- and second-order spectral fits obtained using the I99 SN Ia (W7) yields and Salpeter-IMF-averaged I99 SN cc yields are shown in Figure 1. We also show a close-up view of the fit in the RGS Fe-L bandpass obtained using the T95 SN Ia (W7) and SalpeterIMF-averaged T95 SN cc yields in Figure 2. Figure 2 shows that the T95 SN yields accurately reproduce the RGS spectrum in the Fe-L bandpass.

\subsection{XMM-Newton EPIC Observations of A3112}

We examined the EPIC observations of the cool core cluster A3112 (see Table 1) using version 11.0 of the SAS software. The EPIC data processing and background modeling were carried out with the XMM-Newton Extended Source Analysis Software (XMM-ESAS) and methods (Kuntz \& Snowden 2008; Snowden et al. 2008) described in Paper I. Since the EPIC spectra can constrain $\mathrm{Si}, \mathrm{S}, \mathrm{Fe}$, and $\mathrm{O}$ abundances only in the innermost $30^{\prime \prime}$ region (see Paper I), we examined only the central $30^{\prime \prime}$ spectra in this work. We fit the cluster emission using the absorbed snapec model allowing the absorption column density, $N^{\mathrm{SNe}}, R$, redshift, $k T_{e}$, and normalization parameters freely vary. The best-fit parameters of the instrumental and cosmic $\mathrm{X}$-ray background model components were fixed to the best-fit

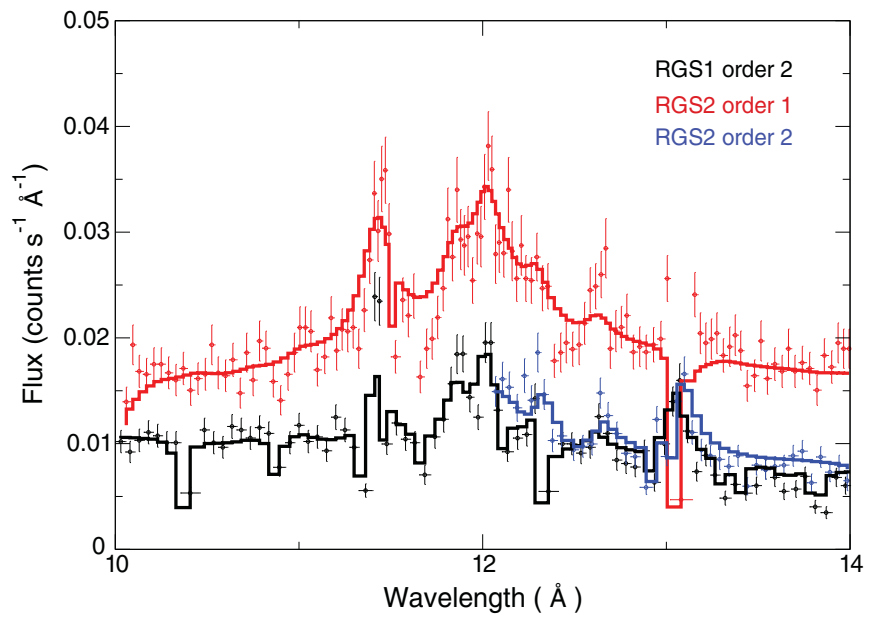

Figure 2. Close-up view of the Fe-L bandpass. The fit was obtained by applying the T95 SN Ia (W7, slow deflagration) and SN cc (weighted over 10-50 $M_{\odot}$ using the Salpeter IMF function) yields to the XMM-Newton RGS spectra. The best-fit model parameters and the goodness of fit are shown in Table 2.

values obtained from the overall fits and reported in Paper I. A conversion factor of 0.41 was again applied to the SNe per $10^{12} M_{\odot}$ derived from EPIC spectral fits to obtain the $N^{\mathrm{SNe}}$ parameter. The best-fit model parameters obtained from the snapec fits to EPIC spectra for various $\mathrm{SN}$ nucleosynthesis models are shown in Table 3 . These final $\chi^{2}$ values, reported in Table 3, were obtained from the best fit determined by $C$-statistics.

The temperature, $\mathrm{SN}$ ratio $(R)$, and the total number of $\mathrm{SN}$ measurements obtained from EPIC spectra are consistent with RGS results (Table 2) at the $1 \sigma$ level. The $1 \sigma$ difference is due to different temperature measurements reported by EPIC and RGS observations. Most of the SN nucleosynthesis models we use in this work produce equally good fits to EPIC data as in the case of RGS analysis. High signal-to-noise CCD resolution EPIC 
Table 3

The Best-fit Parameters of the snapec Model Obtained Using T95, I99, and M10 SN Yields and XMM-Newton EPIC Spectra

\begin{tabular}{|c|c|c|c|c|c|c|}
\hline $\begin{array}{l}\text { SNe Ia } \\
\text { Model }\end{array}$ & $\begin{array}{l}\text { SNe cc } \\
\text { Model }\end{array}$ & $\begin{array}{c}k T_{e} \\
(\mathrm{keV})\end{array}$ & $\begin{array}{c}N^{\mathrm{SNe}} \\
\left(\times 10^{9}\right)\end{array}$ & $R$ & $\begin{array}{l}R_{\%}^{\mathrm{Ia}} \\
(\%)\end{array}$ & $\begin{array}{c}\chi^{2} \\
(1683 \text { dof })\end{array}$ \\
\hline T95 (W7) & T95 & $3.49 \pm 0.02$ & $1.06 \pm 0.02$ & $0.40 \pm 0.01$ & $28.6 \pm 1.0$ & 2737.7 \\
\hline I99 (W70) & I99 & $3.50 \pm 0.02$ & $1.06 \pm 0.02$ & $0.39 \pm 0.01$ & $28.1 \pm 1.0$ & 2723.9 \\
\hline I99 (WDD1) & I99 & $3.47 \pm 0.02$ & $0.82 \pm 0.02$ & $0.76 \pm 0.03$ & $43.2 \pm 2.9$ & 2893.6 \\
\hline I99 (WDD2) & I99 & $3.49 \pm 0.02$ & $0.89 \pm 0.02$ & $0.51 \pm 0.01$ & $33.8 \pm 1.0$ & 2783.9 \\
\hline I99 (CDD1) & I99 & $3.41 \pm 0.01$ & $0.82 \pm 0.11$ & $0.81 \pm 0.03$ & $44.8 \pm 2.9$ & 2927.6 \\
\hline I99 (CDD2) & I99 & $3.50 \pm 0.02$ & $0.87 \pm 0.11$ & $0.49 \pm 0.02$ & $32.9 \pm 1.9$ & 2767.1 \\
\hline M10 (W7) & I99 & $3.51 \pm 0.02$ & $1.06 \pm 0.01$ & $0.40 \pm 0.01$ & $28.6 \pm 1.0$ & 2744.1 \\
\hline M10 (CDEF) & I99 & $3.54 \pm 0.02$ & $1.36 \pm 0.11$ & $0.77 \pm 0.03$ & $43.5 \pm 2.9$ & 2775.8 \\
\hline M10 (CDDT) & I99 & $3.39 \pm 0.02$ & $1.11 \pm 0.10$ & $1.28 \pm 0.07$ & $56.1 \pm 6.5$ & 3627.5 \\
\hline M10 (ODDT) & I99 & $3.49 \pm 0.02$ & $0.83 \pm 0.13$ & $0.82 \pm 0.04$ & $45.1 \pm 3.8$ & 2842.3 \\
\hline
\end{tabular}

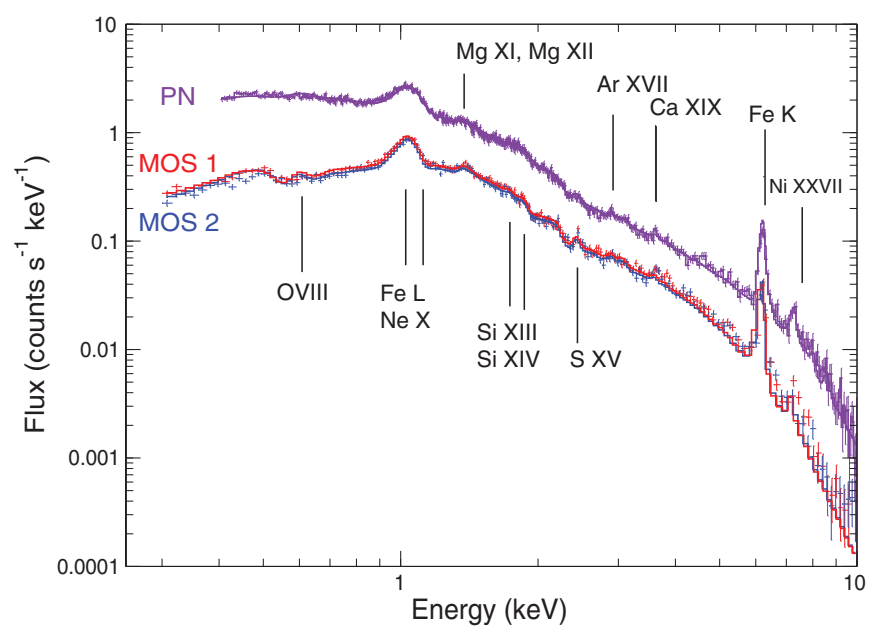

Figure 3. snapec fit to the XMM-Newton MOS1 (red), MOS2 (blue), and PN (magenta) spectra from the innermost $30^{\prime \prime}$ region of A3112 obtained from the observation 0603050101. The background model parameters were fixed to the best-fit values reported by Bulbul et al. (2012). The fit was performed using Iwamoto et al. (1999) W70 SN Ia and Iwamoto et al. (1999) SN cc yields obtained from the weighted average yields over a Salpeter IMF nucleosynthesis yields. The best-fit temperature and $\mathrm{SN}$ ratio $(R)$ and the total number of SN $\left(N^{\mathrm{SNe}}\right)$ parameters, $3.50 \pm 0.02 \mathrm{keV}, 0.39 \pm 0.01$, and $(1.06 \pm 0.02) \times 10^{9}$, respectively, are shown in Table 3.

(A color version of this figure is available in the online journal.)

spectra yield stringent constraints on the model parameters (e.g., $\mathrm{SN}$ ratio $(R)$ and the total number of $\mathrm{SNe}$ ) but cannot distinguish between different types of SN nucleosynthesis models. Maeda et al. (2010) CDDT SN Ia models yield a worse fit to the EPIC spectra compared to Iwamoto et al. (1999), Tsujimoto et al. (1995), or Maeda et al. (2010) W7, CDEF, ODDT SN Ia models.

We find that fits to the EPIC spectra find a slightly wider range of SN type Ia fraction, so that $28.1 \% \pm 1.0 \%$ to $44.8 \% \pm$ $2.9 \%$ of the total SN explosions are SNe Ia for models that use W7, CDD, and WDD nucleosynthesis yields. As in the case of the RGS analysis, the M10 CDEF, CDDT SN Ia models imply a significantly higher fraction of SN Ia explosions $(43.5 \% \pm$ $12.9 \%$ to $56.1 \% \pm 6.5 \%$ ) as a result of lower Fe abundance compared to the W7, W70, CDD, WDD models. We also found that all W7 models from several simulations (e.g., T95, I99, and M10) produce similar goodness of fit, the total number of SNe, and SN Ia fraction. The snapec fits to EPIC MOS1, MOS2, and PN spectra performed using the Iwamoto et al. (1999) W70 SN Ia and Salpeter-IMF-weighted Iwamoto et al. (1999) SN cc metal yields are shown in Figure 3.

\subsection{Astro-H Simulations of A3112}

The Japan/U.S. Astro-H Observatory is scheduled to launch in 2014 and will carry an X-ray calorimeter, the SXS, with a high energy resolution of $\sim 4.5 \mathrm{eV}$ (Takahashi et al. 2010). The detector will be able to determine line widths and metal abundances with high precision, revolutionizing our understanding of physical processes (e.g., turbulence, the contributions of SN explosions to metal enrichment) in the ICM. We simulated $200 \mathrm{ks}$ SXS observations of A3112 using the X-ray events simulator software ( $\operatorname{sim} x 1.2 .1)$, based on the temperature and abundance measurements obtained from the snapec fits convolved with 199 SN Ia (W7) and I99 Salpeter-IMF-weighted SN cc yields. simx uses predefined detector response matrix, effective area, and predicted background for the SXS instrument to produce an event file by convolution with the telescope's point spread function. ${ }^{4}$ The SXS spectrum is extracted using the FTOOL xselect based on the event file produced with the simx software.

We then fit the simulated SXS spectrum with the snapec model convolved with various $\mathrm{SN}$ Ia and $\mathrm{SN}$ cc yields for variable temperature, $N^{\mathrm{SNe}}, R$, redshift, and normalization. Figures 4 and 5 show the soft X-ray band and a close-up view of the Fe-K bandpass for fits to the simulated SXS spectrum of A3112 obtained assuming the same $\mathrm{SN}$ yields used to create the spectrum. The relative contributions of SN Ia and SN cc products to the spectrum are shown as green and blue lines in Figures 4 and 5, respectively. The figures illustrate how the significant amounts of $\mathrm{Fe}$ and $\mathrm{Mg}$ produced by $\mathrm{SN}$ Ia explosions, and large quantities of $\mathrm{O}$ synthesized in SN cc explosions, are manifest in the spectrum. The best-fit parameters and goodness of fits obtained using the I99, T95, and M10 SN yields are shown in Table 4 . The features from metals primarily produced by SN Ia explosions such as $\mathrm{S}, \mathrm{Ar}, \mathrm{Ca}$, and $\mathrm{Ni}$ that were inaccessible to the EPIC and RGS now become important diagnostics for Astro- $H$ data analysis. As a result of the accurate abundance measurements of these metals, uncertainties in the $N^{\mathrm{SNe}}$ and $R$ parameters are reduced, and they may be determined more robustly and with higher precision (see Table 4).

As expected, good fits are found using SN models that have similar elemental yields, e.g., using T95 (W7) or I99 (W70) SN Ia models, to those of the I99 (W7) SN Ia and SN cc yields adopted to produce the simulated Astro- $H$ spectrum (see Table 4). On the other hand, the significantly higher $\chi^{2}$ values obtained from fits with SN models that have markedly different

\footnotetext{
4 http://hea-www.harvard.edu/simx/index.html
} 


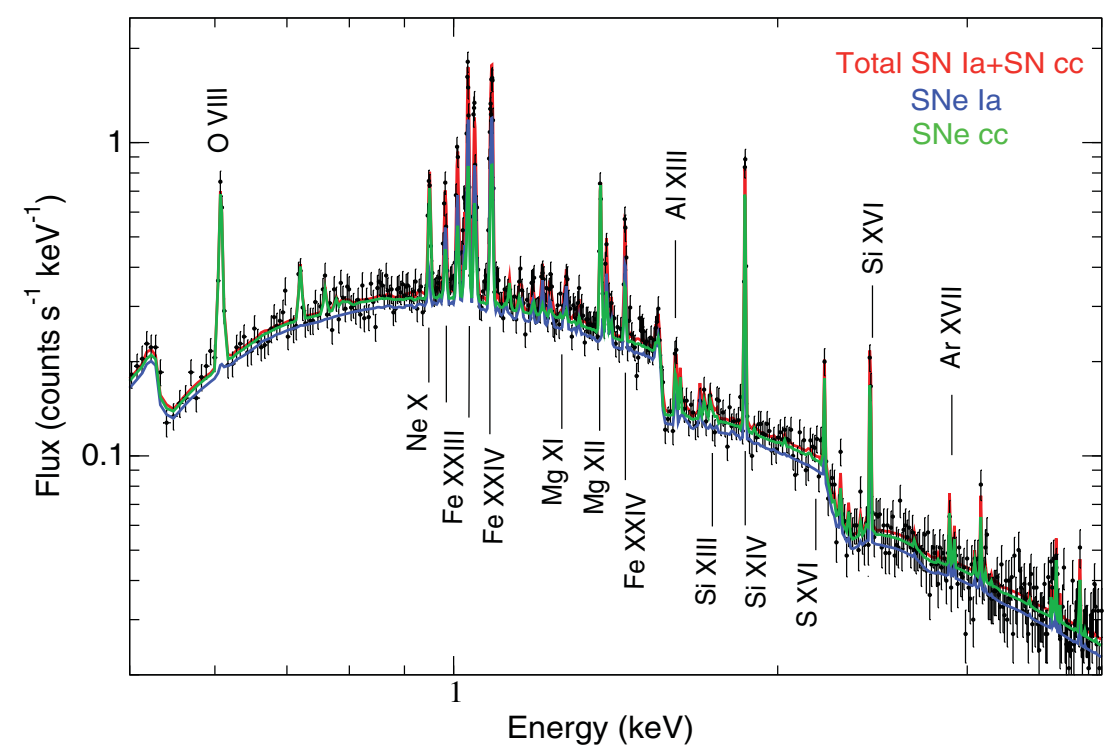

Figure 4. Simulated Astro-H SXS spectra using the snapec model. The fit was performed using Iwamoto et al. (1999) SN Ia yields obtained from W7 models and SN cc yields obtained from the average yield computed using Salpeter IMF weighting (as in Figure 1). The red color shows the best-fit snapec model with T95 W7 SN Ia and SN cc yields. The contribution of SN Ia explosions to the metal abundances are shown in green while the contribution of SN cc explosions are shown in blue.

Table 4

snapec Parameters for the Best Fit to the Simulated Astro-H Spectra of A3112 Assuming I99 SN Ia and SN cc Yields

\begin{tabular}{|c|c|c|c|c|c|}
\hline $\begin{array}{l}\text { SNe Ia } \\
\text { Model }\end{array}$ & $\begin{array}{l}\text { SNe cc } \\
\text { Model }\end{array}$ & $\begin{array}{c}k T_{e} \\
(\mathrm{keV})\end{array}$ & $\begin{array}{l}N^{\mathrm{SNe}} \\
\left(\times 10^{9}\right)\end{array}$ & $R$ & $\begin{array}{c}\chi^{2} \\
(886 \text { dof })\end{array}$ \\
\hline T95 (W7) & T95 & $3.77 \pm 0.03$ & $1.10 \pm 0.07$ & $0.50 \pm 0.02$ & 1124.5 \\
\hline I99 (W7) & I99 & $3.77 \pm 0.03$ & $1.09 \pm 0.06$ & $0.51 \pm 0.02$ & 1119.9 \\
\hline I99 (W70) & I99 & $3.76 \pm 0.03$ & $1.08 \pm 0.06$ & $0.49 \pm 0.02$ & 1113.8 \\
\hline I99 (WDD1) & I99 & $3.78 \pm 0.03$ & $1.06 \pm 0.06$ & $0.60 \pm 0.03$ & 1319.4 \\
\hline I99 (CDD1) & I99 & $3.69 \pm 0.05$ & $1.07 \pm 0.08$ & $0.33 \pm 0.02$ & 1348.9 \\
\hline M10 (CDEF) & I99 & $3.76 \pm 0.03$ & $1.46 \pm 0.08$ & $0.89 \pm 0.05$ & 1223.9 \\
\hline M10 (CDDT) & I99 & $3.83 \pm 0.03$ & $1.16 \pm 0.05$ & $1.54 \pm 0.12$ & 1795.0 \\
\hline M10 (ODDT) & I99 & $3.84 \pm 0.03$ & $1.07 \pm 0.07$ & $0.69 \pm 0.03$ & 1292.6 \\
\hline
\end{tabular}

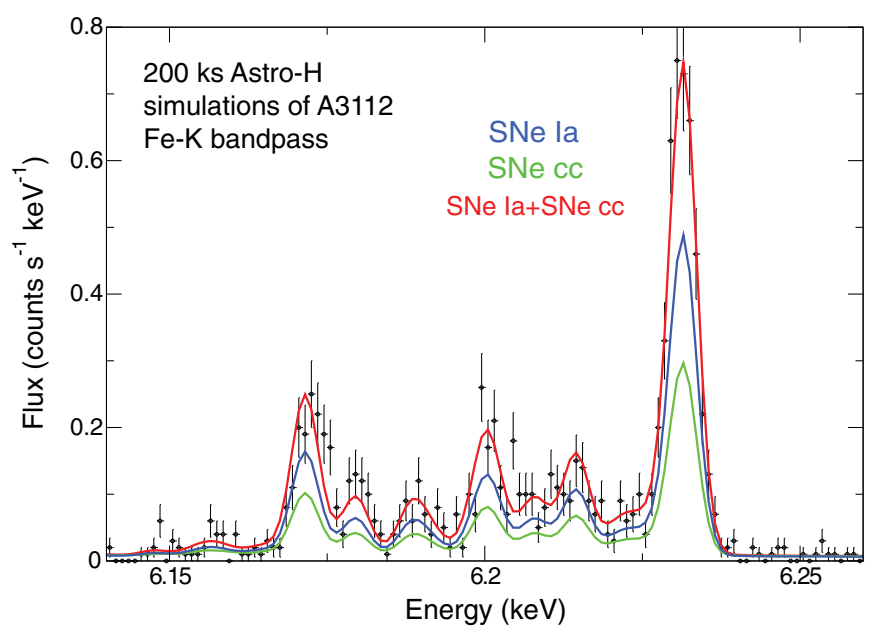

Figure 5. Close-up view of the Fe-K bandpass of the $100 \mathrm{ks}$ simulated Astro- $H$ SXS spectrum, based on the snapec model convolved with I99 SN Ia and SN cc yields. The red color shows the best-fit snapec model for T95 W7 SN Ia and SN cc yields. The contribution of SN Ia explosions to the metal abundances are shown in green while the contribution of SN cc explosions are shown in blue.

yield patterns, e.g., I99 CDD and I99 WDD models, demonstrate that future Astro- $H$ observations of galaxy clusters will allow one to distinguish between different SN models and provide fundamental constraints on SN nucleosynthesis.

\section{DISCUSSION}

By developing and applying a new spectral analysis methodology to RGS spectra, we measure the total number of SNe that have enriched the ICM presently in the inner $52 \mathrm{kpc}$ of A3112 and the relative contributions of SNe Ia and SNe cc. In this section, we compare this with the enrichment expected based on the number of SN Ia and SN cc explosions derived from the amount of starlight and well-established empirically measured SN rates per star formed. We also investigate prospective local enrichment sources related to the brightest cluster galaxy (BCG). In this way, we can interpret our data analysis in the context of the origin of the gas and its metals.

\subsection{Global Cluster Context for the Number of SNe}

Within its virial radius, a sufficiently massive cluster is approximately a closed box. For a global efficiency of converting gas to stars $\varepsilon_{\text {sf }}$, the total mass in stars formed is

$$
M_{* \text { form }}=\varepsilon_{\mathrm{sf}} M_{\mathrm{bary}},
$$

where $M_{\text {bary }}$ is the total baryon mass. At the present time the total mass in stars, whether contained in individual cluster galaxies (including the BCG) or associated with intracluster light (ICL), is

$$
M_{*}=M_{\text {bary }} \varepsilon_{\mathrm{sf}}\left(1-R_{*}\right),
$$


and the mass in gas is

$$
\begin{gathered}
M_{\mathrm{gas}}=M_{\text {bary }}-M_{*} \\
=M_{\text {bary }}\left[1-\varepsilon_{\mathrm{sf}}\left(1-R_{*}\right)\right],
\end{gathered}
$$

where $R_{*}$ is the mass return fraction. We can safely neglect the distinction between the mass in gas and the mass in the ICM for a cluster as rich as A3112 where the gas mass in galaxies is relatively small, and henceforth equate $M_{\text {gas }}$ with $M_{\mathrm{ICM}}$. The star formation efficiency, in terms of the observable $M_{\mathrm{ICM}} / M_{*}$,

$$
\varepsilon_{\mathrm{sf}}=\left(1-R_{*}\right)^{-1}\left(1+M_{\mathrm{ICM}} / M_{*}\right)^{-1}
$$

The total number of SN cc and Type Ia explosions can be expressed as

$$
N^{\mathrm{cc}}=\eta^{\mathrm{cc}} M_{* \text { form }}
$$

and

$$
N^{\text {Ia }}=\eta^{\text {Ia }} M_{* \text { form }},
$$

where $\eta^{\text {cc }}$ and $\eta^{\text {Ia }}$ are, respectively, the specific numbers of SN cc and SN Ia explosions per star formed. Connecting $\eta^{\mathrm{cc}}$ and $\eta^{\mathrm{Ia}}$ to the snapec model parameters defined in Section 2,

$$
R=\frac{\eta^{\mathrm{Ia}}}{\eta^{\mathrm{cc}}}
$$

and

$$
N^{\mathrm{SNe}}=\eta\left(1-R_{*}\right)^{-1} M_{*},
$$

where $\eta=\eta^{\mathrm{II}}+\eta^{\mathrm{Ia}}$. We note that these refer to all of the SNe while in Section $2 ; N^{\mathrm{SNe}}$ and $R$ parameters refer only to $\mathrm{SNe}$ that enrich the ICM.

Adopting a "diet Salpeter" IMF that produces relatively fewer low-mass stars (Bell \& de Jong 2001), Maoz \& Mannucci (2011) estimated $\eta^{\text {Ia }}$ to be $\sim 0.002$. Adopting the mass return fraction and specific SN cc rate for the diet Salpeter IMF: $R_{*} \sim 0.35$ (Fardal et al. 2007; O'Rourke et al. 2011), $\eta^{\text {Ia }} \sim 0.002$, and $\eta^{\mathrm{II}} \sim 0.008$ (Maoz \& Mannucci 2011; Botticella et al. 2012), one predicts $R \sim 0.25$, which corresponds to $R_{\%}^{\mathrm{Ia}}=0.2$, and $\eta \sim 0.01$. For clusters in the A3112 mass range (e.g., $M_{500} \sim 3.0 \times 10^{14} M_{\odot}$, Paper I), recent analysis finds, typically, $M_{*} / M_{\mathrm{ICM}} \sim 0.1$ (Balogh et al. 2011; Lin et al. 2012). Therefore, from Equation (18), the estimated total number of SN explosions per $10^{12} M_{\odot}$ of $\mathrm{ICM}$ is $\sim 1.54 \times 10^{9}$. Multiplying by the correction factor of 0.41 appropriate to the core of A3112, $N^{\mathrm{SNe}}$ becomes $\sim 6.3 \times 10^{8}$. Comparing with our results in Table 2 , we may conclude that the inner $52 \mathrm{kpc}$ of A3112 has been enriched by more $\mathrm{SNe}$, with a higher percentage of $\mathrm{SNe} \mathrm{Ia}$, than is true globally for a typical cluster of its mass.

\subsection{SN Metals Locked Up in Stars}

The galactic mass in clusters is dominated by early-type galaxies that form their stars quickly. This results in the wellestablished enhancement in $[\alpha / \mathrm{Fe}]$, the abundance ratio of $\alpha$-elements to $\mathrm{Fe}$ (expressed as the logarithm with respect to solar)-i.e., SNe cc are preferentially locked up in stars (Loewenstein 2004). In their investigation of the giant elliptical galaxy NGC 4472, Loewenstein \& Davis (2010) found that a ratio of $\mathrm{SNe}$ Ia to total $\mathrm{SNe}$ of $N^{\mathrm{Ia} *} / N^{\mathrm{SNe} *} \sim 0.11$ $\left(N^{\mathrm{cc} *} / N^{\mathrm{SNe} *} \sim 0.89\right)$ and a number of SNe per mass in (presentday) stars of $N^{\mathrm{SNe}} / M_{*} \sim 0.0083$ resulted in $[\alpha / \mathrm{Fe}]_{*} \sim 0.25$ and $Z_{\mathrm{Fe} *} \sim 1$ (as observed in this particular galaxy, but typical of the class) assuming yields from Kobayashi et al. (2006). This enables us to estimate the lock-up corrections, $\eta^{\mathrm{cc} *}$ and $\eta^{\text {Ia* }}$, to the total specific numbers of SNe per star formed. This provides, in turn, the corresponding values available to enrich the ICM, $\eta_{\mathrm{ICM}}^{\mathrm{Ia}}$ and $\eta_{\mathrm{ICM}}^{\mathrm{cc}}$ :

$$
\begin{aligned}
\eta^{\mathrm{Ia} *} & =\left(\frac{N^{\mathrm{Ia} *}}{M_{*}}\right)\left(1-R_{*}\right) \\
& =6.0 \times 10^{-4} Z_{\mathrm{Fe} *},
\end{aligned}
$$

$$
\eta^{\mathrm{cc} *}=\left(\frac{N^{\mathrm{cc} *}}{M_{*}}\right)\left(1-R_{*}\right)
$$

$$
=4.8 \times 10^{-3} Z_{\mathrm{Fe} *},
$$

$$
\eta_{\mathrm{ICM}}^{\mathrm{Ia}}=\eta^{\mathrm{Ia}}-\eta^{\mathrm{Ia} *}=1.4 \times 10^{-3} M_{\odot}^{-1},
$$

and

$$
\eta_{\mathrm{ICM}}^{\mathrm{cc}}=\eta^{\mathrm{cc}}-\eta^{\mathrm{cc} *}=3.2 \times 10^{-3} M_{\odot}^{-1} .
$$

That is, the metal production from $\sim 60 \%$ of $\mathrm{SNe} c \mathrm{c}$, and $\sim 30 \%$ of SNe Ia, must be locked up in stars to enrich them to solar $\mathrm{Fe}$ abundances and $[\alpha / \mathrm{Fe}]_{*} \sim 0.25$. This results in revised predictions of $R \sim 0.30-$ consistent with our RGS results for A3112, but an even lower expected total number of $\mathrm{SNe}-N^{\mathrm{SNe}} \sim 2.9 \times 10^{8} \mathrm{SNe}$.

\subsection{Direct SNe Injection}

Sand et al. (2012) recently estimated a specific SN Ia rate in cluster galaxies of $r_{\mathrm{SNI}} \sim 0.04 \mathrm{SNuM}^{5}$ within $R_{200}(\sim 1 \mathrm{Mpc})$ (see also Maoz et al. 2010) that may be directly injected into the ICM by the $r<52 \mathrm{kpc}$ stellar population. The implied level of SNIa available for enrichment accumulated over time $\tau$ is

$$
N^{\mathrm{Ia}}=10^{-4} M_{*}(<52 \mathrm{kpc})\left(\frac{r_{\mathrm{SNIa}}}{0.1 \mathrm{SNuM}}\right)\left(\frac{\tau}{10^{9} \mathrm{yr}}\right),
$$

where the stellar mass $M_{*}$ refers to the $r<52 \mathrm{kpc}$ region, and $r_{\text {SNIa }}$ should now be interpreted as an average over $\tau$. We use the Hernquist approximation (Hernquist 1990) to deVaucouleurs profiles for the A3112 BCG and ICL components and estimate $M_{*}=3.6 \times 10^{11} M_{\odot}$ within $52 \mathrm{kpc}$-i.e., $M_{*}=0.88 M_{\mathrm{ICM}}$. A large star-to-gas ratio, $M_{*} \sim M_{\mathrm{ICM}}$, and a long timescale $\tau>10^{9} \mathrm{yr}$ (with the accompanying increase in $r_{\text {SNIa }}$ ) would be required for this to be a significant source of enrichment.

\subsection{The Cluster Enrichment Puzzle as Realized in the Core of $A 3112$}

For most of the yield sets we consider, the level of ICM enrichment for the central $(4.06 \pm 0.70) \times 10^{11} M_{\odot}$ requires $\sim 1.1 \times 10^{9} \mathrm{SNe}$, with $\mathrm{SNe}$ Ia accounting for $\sim$ one-third of the total. Although our SN parameters are derived for the inner $52 \mathrm{kpc}$, the high gas mass and temperature imply that this gas is primarily intracluster in origin. If we account for the fraction of SN products locked up in cluster galaxy stars, which is higher for SNe cc based on the measured stellar $[\alpha / \mathrm{Fe}]$ enhancement, we can explain the proportions of $\mathrm{SNe}$ Ia and $\mathrm{SNe}$ cc that we infer enriched the ICM. However, the total number of $\mathrm{SNe}$ we infer is greater than expected by a factor of $\sim 4$ for

\footnotetext{
$51 \mathrm{SNuM}=$ Supernova rate per $100 \mathrm{yr}$ per $10^{10} M_{\odot}$ in stars.
} 
a typical star-to-gas ratio, $M_{*} / M_{\mathrm{ICM}} \sim 0.1$. These conclusions are altered by direct injection of SN Ia ejecta in the BCG only if accumulated over a large fraction of a Hubble time and are exacerbated for the M10 CDEF and CDDT models. Evidently, the stellar population responsible for enriching this material is characterized by enhanced efficiency in producing $\mathrm{SNe}$, and/or the mass in stars responsible for the enrichment, $M_{*}>0.3 M_{\mathrm{ICM}}$.

Larger estimates of $M_{*} / M_{\mathrm{ICM}} \sim 0.2$ at the A3112 mass range may be found in Gonzalez et al. (2007; see also Langaná et al. 2011). Indeed A3112 was investigated by Gonzalez et al. (2007), from which we may derive $M_{*} / M_{\mathrm{ICM}} \sim 0.27$. The implication is that there is substantial ICL component unaccounted for in other studies (see also Bregman et al. 2010) and that the star formation efficiency is very high (Equation (14)).

Even if of intracluster origin, the enrichment of this inner cluster gas is likely to reflect the somewhat special conditions in the cluster core where high initial overdensity may result in enhanced efficiency of galaxy and star formation. Thus, the effective value of $M_{*} / M_{\mathrm{ICM}}$ may very well be larger than is representative of the ICM as a whole. At the same time, for rich galaxy clusters in general, the global ICM shows enrichment beyond what is expected based on the stars we see in galaxies today (Portinari et al. 2004; Loewenstein 2006; Maoz et al. 2010; Bregman et al. 2010).

Another possible mechanism contributing to the greater than expected number of $\mathrm{SNe}$ found in our fits is strong early enrichment of the lowest-entropy X-ray-emitting gas stripped from early galaxies. The heavier metals found in gas stripped at large radii will settle into the cluster core due to the central gravitational potential, while the stars that produced them remain at larger radii. Therefore, a significant fraction of metals residing in the cluster core that we observe today may not have originated from the local stellar population (Million et al. 2011). In this scenario, the apparent total expected number of $\mathrm{SNe}$ will be enhanced relative to that inferred from $M_{*} / M_{\mathrm{ICM}}$. A more extensive study of a larger sample is needed to fully address this issue.

\section{CONCLUSIONS}

In this work we introduce a new approach to determine the total number of SN explosions and the relative contribution of $\mathrm{SNe}$ Ia to the total enrichment of the ICM using high-resolution $\mathrm{X}$-ray observations of clusters of galaxies. The method has been implemented as an XSPEC model called snapec. The method relies upon the apec model for spectral emission, but uses the metal abundances determined from $\mathrm{SN}$ yields from the latest published SN Ia and SN cc explosion models normalized to the solar abundances (e.g., Anders \& Grevesse 1989; Lodders 2003, or Asplund et al. 2009). This model returns the total number of $\mathrm{SN}$ explosions and the relative contribution of the SN types in the spectral extraction region that enrich the ICM integrated over the cluster life-time, although it does not explicitly constrain the $\mathrm{SN}$ products locked up in stars in member galaxies. Contrary to previous studies in the literature, this method has the ability to use all relevant elements, allowing spectra and SN yields obtained from published models to determine the best fit to their abundances. The main advantage of this approach is that it provides a self-consistent single set of uncertainties on the total number of $\mathrm{SN}$ explosions and relative fraction of $\mathrm{SN}$ types by directly allowing these parameters to be determined by SN yields provided by simulations. This method provides both rigorous assessments of uncertainty and improved statistical efficiency in estimating the total number of $\mathrm{SN}$ explosions and relative $\mathrm{SN}$ fractions.

We use XMM-Newton EPIC and RGS observations of A3112 to validate the method and determine the total number of $\mathrm{SNe}$ and fraction of SNe Ia using various published SN yields (Tsujimoto et al. 1995; Iwamoto et al. 1999; Maeda et al. 2010). Since the T95, I99, and M10 SN models produce equally good fits to RGS data, current CCD resolution EPIC and highresolution RGS observations of clusters of galaxies do not allow one to distinguish between different SN models. The SN Ia fraction reported by EPIC observations are consistent with RGS measurements at the $1 \sigma$ level. We also find that the T95, I99, and M10 models produce consistent values of the total number of SNe and SN Ia fractions. $30.3 \% \pm 5.4 \%$ to $37.1 \% \pm 7.1 \%$ of the total $\mathrm{SN}$ explosions are $\mathrm{SNe}$ Ia and the total number of $\mathrm{SN}$ explosions are in the range of $(1.06 \pm 0.34) \times 10^{9}$ to $(1.28 \pm 0.43) \times 10^{9}$. The best-fit SN Ia ratios for WDD and CDD we obtained from RGS spectra are consistent with de Plaa et al. (2007) measurements. However, de Plaa et al. (2007) find a lower SN Ia fraction for W7 SN Ia models.

We also applied this method to simulated Astro- $H$ SXS observations of A3112. Astro- $H$ simulations were performed using I99 W7 SN Ia and I99 Salpeter-weighted SN cc models. Assuming that $199 \mathrm{~W} 7$ models reflects the true nature of the ICM enrichment, higher $\chi^{2}$ values obtained from the I99 WDD, I99 CDD, and M10 CDDT models indicate that we will be able to distinguish between different SN models with Astro- $H$ observations of clusters of galaxies.

The estimated mix of SNe Ia and SNe cc $(30 \%$ and $70 \%$, respectively) expected in the ICM of A3112 based on wellestablished, empirically measured $\mathrm{SN}$ rates per star formed is close to what we infer to have enriched the ICM in the inner $52 \mathrm{kpc}$ of A3112 based on this new method of high-resolution spectral analysis. The inferred level of SN enrichment, $\sim 2.7 \times$ $10^{9} \mathrm{SNe}$ per $10^{12} M_{\odot}^{-1}$ of ICM, corresponds to $M_{*} / M_{\mathrm{ICM}} \sim$ 0.3-0.4-compared to $M_{*} / M_{\mathrm{ICM}} \sim 0.1$, which has been estimated as typical for clusters in the A3112 mass range.

When applied to RGS observations of a large sample of cool core galaxy clusters, this method can provide stringent constraints on the contribution of SN explosions to the metal enrichment of the ICM and the evolution of the total number of $\mathrm{SNe}$ Ia with redshift. This method will also provide an independent measure of a key diagnostic of the nature of SN Ia progenitors, the dependence of the evolution of SN Ia rate to the distribution of the delay time between the formation of the progenitor system and its explosion as an $\mathrm{SN}$.

The authors thank Hiroya Yamaguchi for kindly providing help on the $\operatorname{sim} x$ software. We also thank the referee for their careful reading of the paper and insightful suggestions. We gratefully acknowledge support for this research from NASA XMM-Newton grant NNX09AP92G and NASA ROSES-ADP grant NNX09AC71G.

\section{REFERENCES}

Anders, E., \& Grevesse, N. 1989, Geochim. Cosmochim. Acta, 53, 197 Asplund, M., Grevesse, N., Sauval, A. J., \& Scott, P. 2009, ARA\&A, 47, 481 Balogh, M. L., Mazzotta, P., Bower, R. G., et al. 2011, MNRAS, 412, 947 Barbary, K., Aldering, G., Amanullah, R., et al. 2012, ApJ, 745, 32

Baumgartner, W. H., Loewenstein, M., Horner, D. J., \& Mushotzky, R. F. 2005, ApJ, 620, 680

Bell, E. F., \& de Jong, R. S. 2001, ApJ, 550, 212

Botticella, M. T., Smartt, S. J., Kennicutt, R. C., et al. 2012, A\&A, 537, A132 Bregman, J. N., Anderson, M. E., \& Dai, X. 2010, ApJ, 716, L63 
Bulbul, G. E., Hasler, N., Bonamente, M., \& Joy, M. 2010, ApJ, 720, 1038

Bulbul, G. E., Smith, R. K., Foster, A., et al. 2012, ApJ, 747, 32

de Plaa, J., Werner, N., Bleeker, J. A. M., et al. 2007, A\&A, 465, 345

De Young, D. S. 1978, ApJ, 223, 47

Dupke, R. A., \& Arnaud, K. A. 2001, ApJ, 548, 141

Dupke, R. A., \& White, R. E., III. 2000, ApJ, 537, 123

Fardal, M. A., Katz, N., Weinberg, D. H., \& Davé, R. 2007, MNRAS, 379, 985

Gal-Yam, A., Maoz, D., \& Sharon, K. 2002, MNRAS, 332, 37

Gonzalez, A., Zaritsky, D., \& Zabludoff, A. I. 2007, ApJ, 666, 147

Grange, Y. G., de Plaa, J., Kaastra, J. S., et al. 2011, A\&A, 531, A15

Gunn, J. E., \& Gott, J. R., III. 1972, ApJ, 176, 1

Hernquist, L. 1990, ApJ, 356, 359

Iwamoto, K., Brachwitz, F., Nomoto, K., et al. 1999, ApJS, 125, 439

Izotov, Y. I., \& Thuan, T. X. 2010, ApJ, 710, L67

Kalberla, P. M. W., Burton, W. B., Hartmann, D., et al. 2005, A\&A, 440, 775

Kobayashi, C., Umeda, H., Nomoto, K., Tominaga, N., \& Ohkubo, T. 2006, ApJ, 653,1145

Kuntz, K. D., \& Snowden, S. L. 2008, A\&A, 478, 575

Langaná, T. F., Zhang, Y.-Y., Reiprich, T. H., \& Schneider, P. 2011, ApJ, 743, 13

Lin, Y.-T., Stanford, S. A., Eisenhardt, P. R. M., et al. 2012, ApJ, 745, L3

Lodders, K. 2003, ApJ, 591, 1220

Loewenstein, M. 2004, in Origin and Evolution of the Elements, ed. A.

McWilliam \& M. Rauch (Cambridge: Cambridge Univ. Press), 422

Loewenstein, M. 2006, ApJ, 648, 230

Loewenstein, M., \& Davis, D. S. 2010, ApJ, 716, 384

Maeda, K., Röpke, F. K., Fink, M., et al. 2010, ApJ, 712, 624

Maoz, D., \& Mannucci, F. 2011, arXiv:1111.4492
Maoz, D., Sharon, K., \& Gal-Yam, A. 2010, ApJ, 722, 1879

Markevitch, M. 2007, arXiv:0705.3289

Mathews, W. G., \& Baker, J. C. 1971, ApJ, 170, 241

Matsushita, K., Böhringer, H., Takahashi, I., \& Ikebe, Y. 2007, A\&A, 462, 953

Million, E. T., Werner, N., Simionescu, A., \& Allen, S. W. 2011, MNRAS, 418, 2744

Mushotzky, R. F., \& Loewenstein, M. 1997, ApJ, 481, L63

Nomoto, K., Tominaga, N., Umeda, H., Kobayashi, C., \& Maeda, K. 2006, Nucl. Phys. A, 777, 424

O'Rourke, D. J. P., Shabala, S. S., \& Alexander, P. 2011, MNRAS, 418, 2145

Perlmutter, S., Aldering, G., Goldhaber, G., et al. 1999, ApJ, 517, 565

Portinari, L., Moretti, A., Chiosi, C., \& Sommer-Larsen, J. 2004, ApJ, 604, 579

Riess, A. G., Filippenko, A. V., Challis, P., et al. 1998, AJ, 116, 1009

Ruiz-Lapuente, P., \& Canal, R. 1998, ApJ, 497, L57

Salpeter, E. E. 1955, ApJ, 121, 161

Sand, D. J., Graham, M. L., Bildfell, C., et al. 2012, ApJ, 746, 163

Sato, K., Tokoi, K., Matsushita, K., et al. 2007, ApJ, 667, L41

Simionescu, A., Werner, N., Böhringer, H., et al. 2009, A\&A, 493, 409

Smith, R. K., Brickhouse, N. S., Liedahl, D. A., \& Raymond, J. C. 2001, ApJ, 556, L91

Snowden, S. L., Mushotzky, R. F., Kuntz, K. D., \& Davis, D. S. 2008, A\&A, 478,615

Takahashi, T., Mitsuda, K., Kelley, R., et al. 2010, Proc. SPIE, 7732, 27

Tsujimoto, T., Nomoto, K., Yoshii, Y., et al. 1995, MNRAS, 277, 945

Werner, N., Böhringer, H., Kaastra, J. S., et al. 2006a, A\&A, 459, 353

Werner, N., de Plaa, J., Kaastra, J. S., et al. 2006b, A\&A, 449, 475

Woosley, S. E., \& Weaver, T. A. 1995, ApJS, 101, 181

Yungelson, L. R., \& Livio, M. 2000, ApJ, 528, 108 